\section{MS1-04 Sulfur SAD phasing at the Photon Factory macromolecular crystallography beamlines in Japan}

Yusuke Yamada ${ }^{1,2}$, Naohiro Matsugaki ${ }^{1,2}$, Dorothee Liebschner ${ }^{1}$, Masahiko Hiraki ${ }^{2,3}$, Ayaka Harada ${ }^{1,2}$, Miki Senda ${ }^{1,2}$, Hisayoshi Makio $^{1}$, Toshiya Senda ${ }^{1,2}$

1. Structural Biology Research Center and Photon Factory, Institute of Materials Structure Science, High Energy Accelerator Research Organization (KEK)

2. School of High Energy Accelerator Science, The Graduate University for Advanced Studies (SOKENDAI)

3. Mechanical Engineering Center, High Energy Accelerator Research Organization (KEK)

\section{email: yusuke.yamada@kek.jp}

Sulfur SAD phasing is a method to determine phases by measuring anomalous signals from light atoms such as sulfur and phosphor, which are naturally contained in the most of macromolecular crystals. This method has an advantage that any heave-atom substitutions are not necessary for phase determination. Structural Biology Research Center in KEK has promoted this promising phasing technique to user community by developing a dedicated beamline and peripheral technologies such as sample mounting methods and automated data analysis pipeline. BL-1A is one of the five macromolecular crystallography beamlines at the Photon Factory (PF), and has been operated since 2010. The beamline was designed from the beginning to take full advantage of $\mathrm{X}$-ray beams at around $3 \AA$ aiming to explore the applicability of long wavelength for sulfur SAD phasing. The beam was highly focused at the sample to reduce unwanted scattering from the solvent around microcrystals. The available long wavelength of BL-1A is ranging from 2.7 to $3.5 \AA$. The advantage of larger anomalous signals from light atoms can be easily spoiled by severe absorption of the beam by air around the sample, which is one of the practical difficulties in performing accurate SAD experiment with longer wavelength. To mitigate the absorption effects, a bulky standing chamber was recently introduced in order to operate the whole diffractometer system in helium, where a helium cold stream is continuously supplied and recycled. A dedicated sample changer was developed to minimize the contamination of air during the exchange process. The setup allows simple beamline operation thanks to the experimental environment common at any available wavelength. PReMo is an experimental data management system in the PF macromolecular crystallography beamlines. This also has a capability to process a diffraction data set collected at the beamlines and proceed to further analysis as a data analysis pipeline. In the presentation, an overview of the current status and future plan of the beamline and peripheral technology developments will be given. De-novo structural solutions by sulfur SAD phasing at the beamline are shown as well as methodological studies performed with various wavelengths to discuss the feasibility of extending the wavelength range.

Keywords: sulfur SAD, beamline, macromolecular crystallography

\section{MS1-O5 3-D Data Collection strategy accounting radiation damage}

Alexander N. Popov ${ }^{1}$

1. ESRF, BP-220, Grenoble, 38043, France

email: apopov@esrf.fr

Use of micro-beams for diffraction data collection from the often in-homogeneously diffracting crystals of macromolecules allows improving the ratio of measured signal to noise and increases achievable resolution. In order utilize the advantages of micro-beam conditions, all available material of a crystal must to be used for measurements thus reducing the inevitable radiation damage effects. For this purpose, the sample during or between X-ray exposures is translated (e.g. helical or multi-positional data collection strategies). The optimum choice of crystal displacements and data collection parameters obviously should be defined by accounting the shape and the sizes of a crystal. Here we would like to present next step in the development of the program BEST [Bourenkov, G.P. \& Popov, A.N. (2010). Acta Cryst. D66, 409-419. ] intended for optimal planning of data collection. The new version of the program is based on the use of approximate 3D model of a crystal. Diffraction intensity at any moment of data collection is calculated as the sum of diffraction intensities from crystal voxels taking into account the profile of primary $\mathrm{X}$-ray beam and the dose absorbed by each voxel. Such modelling significantly improves the accuracy of predicted data statistics (also for data collection involving rotation without translations), and allows selecting the best trajectory of the crystal movements.

Keywords: BEST, radiation damage, data collection 